Z. klin. Chem. u. klin. Biochem.

7. Jg., S. $576-580$, November 1969

\title{
Carboxypeptidasen im Serum bei entzündlichen Erkránkungen
}

\author{
Von W. Appel, H. Hermann und E. Huth
}

\begin{abstract}
Aus der Biocbemischen Abteilung des Forscbungsinstitutes der Firma Knoll A.-G., Chemische Fabriken, Ludivigsbafen/Rhein (Direktor: Prof. Dr. E. Biekert) und der Kinderklinik des Klinikum Mannbeim der Universität Heidelberg (Direktor: Prof. Dr. E. Hutb)
\end{abstract}

(Eingegangen am 28. Mai 1969)

\author{
Herrn Professor Dr. Dr. Volker Klingmüller anläßlich seines 60. Geburtstages genvidnet
}

\begin{abstract}
Die Seren von 91 Kindern im Alter von 2-13 Jahren wurden untersucht. Darunter befanden sich 21 gesunde Kinder, die anderen hatten vorwiegend entzündliche Erkrankungen. Quantitativ mit Hilfe von Mikrolitermethoden bestimmt wurden die Blutkörperchensenkungsgeschwindigkeit, die absolute Leukocytenzahl, die Aktivitäten der sog. „Leucinaminopeptidase“ (Arylamidase, LAP) und die $\operatorname{der}$ Carboxypeptidase A. L-Leucin-p-nitranilid (Leupa), $\mathrm{N}_{\alpha}$-Carbonaphthoxy-L-phenylalanin (CNP) und $\mathrm{N}_{\alpha}$-Carbobenzoxyglycyl-L-phenylalanin (Z-Gly-Phe) dienten als Substrate. Zwischen den beiden Enzymen besteht keine Korrelation, ebensowenig überraschenderweise auch zwischen den Aktivitäten der Carboxypeptidase(n) gegenüber den beiden als spezifisch geltenden Substraten, was zur Annahme einer neuen Enzymgruppe zwingt. Bei entzündlichen Prozessen sowie bei 2 von 4 Leukämien ist die Carboxypeptidase A (Substrat CNP) erhöht,' am höchsten bei bakteriellen Infektionen, z. B. Pneumonien. Die Carboxypeptidase A (Substrat Z-Gly-Phe) und die Leucinaminopeptidase bleiben im Normalbereich. Diese Erhöhung der Enzymaktivität geht statistisch gesichert bei allen hochakuten Entzündungen parallel der Blutkörperchensenkungsgeschwindigkeit und der Leukocytenzahl. Beginnende und vor allem abklingende Formen weisen zwar ebenfalls erhöhte Blutkörperchensenkungsgeschwindigkeit und Leukocytenzahl auf, die Enzymaktivität ist jedoch nur geringfügig oder nicht erhöht. Die Brauchbarkeit des Substrates CNP zur Bestimmung der Carboxypeptidase für diagnostische Zwecke, besonders bei entzündlichen Krankheitsformen, wird diskutiert. Über Methodik und Enzymverteilungsmuster von Peptidasen und Proteinasen im Serum von Kindern wird an anderer Stelle berichtet.
\end{abstract}

\section{Serun carboxypeptidases in inflammatory illnesses}

Sera were studied from 91 children in the age range 2-13 years. 21 were healthy and the remainder were suffering from chiefly inflammatory illnesses. The erythrocyte sedimentation rate, the absolute leucocyte count and the activity of "leucine aminopeptidase" (arylamidase, LAP) and of carboxypeptidase $A$ were determined by microlitre methods. L-leucine- $p$-nitranilide (Leupa), $N_{\alpha}$-carbonaphthoxyL-phenylalanine (CNP) and $\mathrm{N}_{\alpha}$-carbobenzoxyglycyl-L-phenylalanine (Z-Gly-Phe) were used as substrates. There was no correlation between the two enzyme activities. Surprisingly, there was also no correlation between the two carboxypeptidases, measured against either of the specific substrates, which suggests a new group of enzymes. In inflammatory conditions and in 2 out of 4 leukaemias, carboxypeptidase A (substrate CNP) was increased, showing the highest levels in bacterial infection, e. g., pneumonia. Carboxypeptidase A (substrate Z-Gly-Phe) and leucine aminopeptidase remained normal. In all highly acute inflammatory conditions, this increase in enzyme activity is statistically significant and is parallelled by the erythrocyte sedimentation rate and leucocyte count. In the early stages and especially when the condition is declining, the erythrocyte sedimentation tate and leucocyte count are increased, but the enzyme activity is only slightly increased or not at all. The use of CNP for the determination of carboxypeptidase for diagnostic purposes, especially inflammatory conditions, is discussed. The methodology and the pattern of distribution of peptidases and proteinases in the serum of children will be reported elsewhere.

Durch die Erkenntnis der Bedeutung der Lysosomen für proteolytische Prozesse (1) wurde die Frage nach deren Beteiligung am Entzündungsgeschehen wieder aktuell. Uns interessierten die Leukocyten als Lysosomenträger (2). Ausgehend von Seren mit einem hohen Leukocytengehalt, u. a. leukämischen Seren, sollte geprüft werden, inwieweit eine Erhöhung der Aktivität proteolytischer Enzyme meßbar ist. In einer Screeningstudie untersuchten wir erstmalig systematisch das Peptidasen- und Proteasenmuster im Serum unter Ausklammerung der proteolytischen Enzyme des Gerinnungssystems. Die Ergebnisse werden an anderer Stelle veröffentlicht werden (3). Aus dieser Studie ergaben sich Anhaltspunkte für die Beteiligung von Exopeptidasen. Wir untersuchten daher die Gesamtaktivitäten der „Leucinaminopeptidase“ (Aminosäurearylamidasen) und der Carboxypeptidasen parallel mit der Blutkörperchensenkungsgeschwindigkeit und der Leukocytenzahl. Auf Grund weiterer Voruntersuchungen wählten wir zur Bestimmung der „Leucinaminopeptidase“ (LAP) nur das chromogene Substrat L-Leucin- $p$-Nitranilid (Leupa), mit dem jedoch - streng genommen - nur die Aktivität der neutralen Aminosäurearylamidasen (ËC 3.4.1 ...) erfaßt wird. Für die Bestimmung der Carboxypeptidase A (EC 3.4.1.2) benutzten wir dagegen parallel das Peptidsubstrat $\mathrm{N}_{\alpha}$-Carbobenzoxyglýcyl-L-phenylalanin (Z-Gly-Phe) und das chromogene Substrat $\mathrm{N}_{\alpha^{-}}$ Carbonaphthoxyphenylalanin (CNP).

Um unsere Patienten möglichst wenig zu belasten, wurde, insbesondere bei Kleinkindern, die Anwendung von teilweise neu ausgearbeiteten Mikrolitermethoden (4) erforderlich.

\section{Methoden und Material}

Die Bestimmung der Aminosäurearylamidasen erfolgte nach (5) mittels der Testkombination „Boehringer“, die der Carboxypeptidase A mit Z-Gly-Phe nach (6), mit CNP nach (7) (zit. in 8), jeweils im Mikrolitersystem mit 20 bzw. 50 bzw. $10 \mu l$ Serum (4) $)^{1}$.

1) Die genannten synthetischen Substrate und Eichsubstanzen sind im Handel erhältlich. 
Die Blutkörperchensenkungsgeschwindigkeit nach 2 Stdn. und die absolute Leukocytenzahl wurden nach den üblichen Methoden ermittelt. Die Blutproben wurden unmittelbar nach der Entnahme zu Serum verarbeitet und spätestens nach 3 Stdn. der Enzymbestimmung zugeführt. Insgesamt wurden 91 Kinder untersucht, die - zur Erhöhung der Allgemeingültigkeit der erhaltenen Ergebnisse - in zwei Kollektive auf zwei Jahre verteilt wurden: Kollektiv I umfaßte den Zeitraum zwischen Mai und Juli 1967 (40 Kinder), Kollektiv II den zwischen April und Mai 1968 (51 Kinder). Die Analysen erfolgten mit den Mikrolitersystemen „Beckman-Spinco“ (Fa. Beckman Instr., München) und „Eppendorf" (Fa. Netheler u. Hinz, Hamburg). Bei den Entzündungen handelt es sich zum größten Teil um "frische“ Entzündungen. Zwar war eine strenge Trennung zwischen bakteriellen und viralen Erkrankungen nur schwer durchführbar, da in den meisten Fällen keine Erreger nachgewiesen wurden; sie wurde trotzdem versucht. Die statistische Auswertung exfolgte mittels des t-Tests nach Student und der Regressionsanalyse mit Hilfe einer elektronischen Datenverarbeitungsanlage IBM 1440.

\section{Ergebnisse}

\section{Aufscbliisselung der Kollektive}

\section{Kollektiv I}

Chronische myeloische Leukämie $(n=3)$

frische Pneumonie $(n=3)$

Virus-Infekt $(n=4)$

Bronchitis $(\mathrm{n}=2)$

Gastroenteritis ( $n=2)$

verschiedene bakterielle Entzündungen $(n=4)$

Colitis ulcerosa $(n=1)$

abheilende Entzündungen $(n=6)$

Diabetes mellitus $(n=2)$

gesunde Kinder $(n=10)$.

\section{Kollektiv II}

Gruppe A: Akute lymphatische Leukämie $(n=3)$; Reticulose $(\mathrm{n}=1)$

Gruppe B : fieberhafte Virus-Infekte $(n=13)$; grippale Infekte, Pseudocroup; seröse Meningitis, Lymphadenitis)

Gruppe C: frische Pneumonie ( $n=4)$

Gruppe D: gastrointestinale Entzündungen $(n=3)$

Gruppe E: Bronchitis ( $\mathrm{n}=1$ )

Gruppe F: verschiedene bakterielle Entzündungen $(\mathrm{n}=8)$

Gruppe G: theumatische fieberhafte Entzündungen $(\mathrm{n}=2)$

Gruppe $H$ : abheilende Entzündung verschiedener Genese ( $n=5)$

Gruppe J: Diabetes mellitus $(n=6)$

Gruppe K: gesunde Kinder $(n=11)$.

\section{Normalbereiche}

Die Absolutwerte der Enzymaktivitäten, insbesondere auch die Normalbereiche sind bei beiden Kollektiven praktisch identisch. Es wurden folgende Normalwerte bei 21 gesunden Kindern gefunden:

Carboxypeptidase A (CNP):

$$
\overline{\mathbf{x}}=2,8 \mathrm{mU} / \mathrm{m} l ; \mathrm{V}=11,5 \%
$$

Carboxypeptidase A (Z-Gly-Phe):

$$
\overline{\mathbf{x}}=106,8 \mathrm{mU} / \mathrm{m} l ; \mathrm{V}=61,2 \%
$$

Leucinaminopeptidase:

$$
\overline{\mathbf{x}}=22,1 \mathrm{mU} / \mathrm{ml} ; \mathrm{V}=32,2 \%
$$

\section{Altersabbängigkeit}

Die Kinder waren zwischen 1 und 13 Jahre alt. Für alle drei Enzymaktivitäten lassen sich keine Beziehungen zum Lebensalter erkennen. Dies gilt für Gesunde und Kranke.

\section{Abbängigkeit vom Krankbeitstyp}

Die Abbildungen $1-3$ zeigen die Enzymaktivitäten von Carboxypeptidase A gegenüber CNP und Z-Gly-Phe sowie von Leucinaminopeptidase, aufgeteilt in die Gruppen $\mathrm{A}-\mathrm{K}$ des Kollektives II (s. o.).

Die höchsten Enzymaktivitäten weisen die beiden Fälle von akuter lymphatischer Leukämie, die fieberhaften Virus-Infekte sowie eine frische Pneumonie auf. Bronchitis, gastrointestinale und sonstige bakterielle Entzündungen liegen etwas niedriger. Die rheumatischen „fieberhaften" Entzündungen zeigen die geringste Aktivität. Die abheilenden Entzündungen liegen nur noch

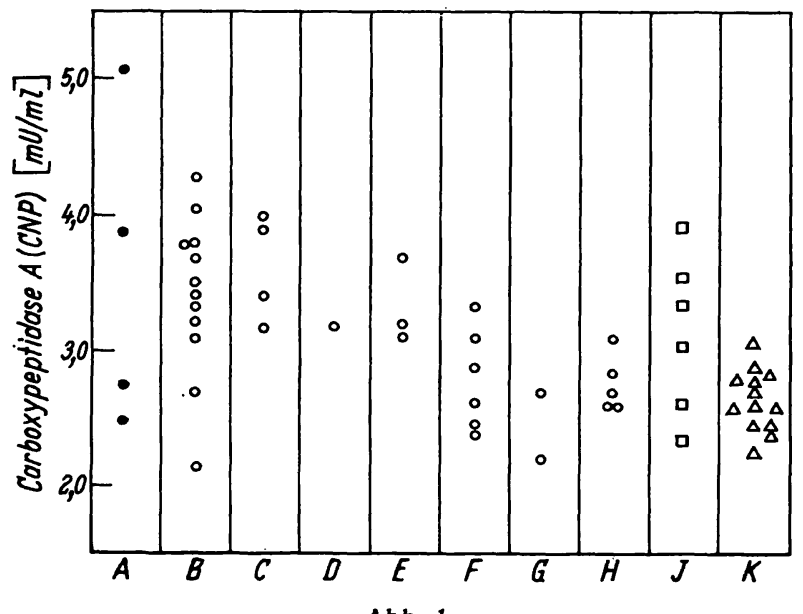

Aktivität der Serum-Carboxypeptidase A gegenüber CNP, aufgeteilt nach Krankheitsbildern. Gruppen $A-K$ s. Text

- = Akute lymphatische Leukämie, Reticulose $\circ=$ Entzündliche Erkrankungen

$\stackrel{\square=\text { Diabetes mellitus }}{\Delta}=$ Gesunde Kinder

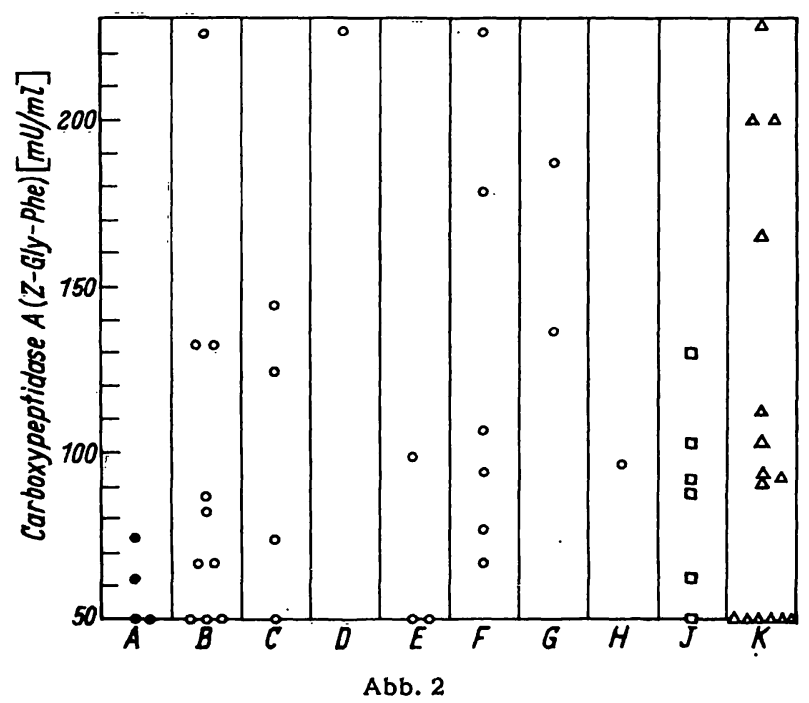

Aktivität der Serum-Carboxypeptidase A gegenüber Z-Gly-Phe, aufgeteilt nach Krankheitsbildern. Gruppen A-K s. Text - = Akute lymphatische Leukämie, Reticulose - Entzündliche Erkrankungen $\square=$ Diabetes mellitus 


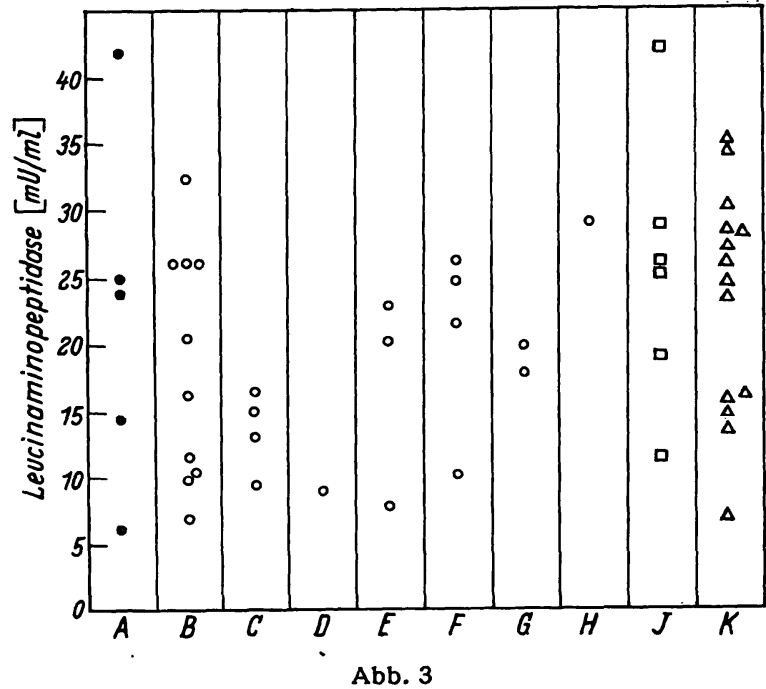

Aktivität der Serum-,,Leucinaminopeptidase" gegenüber Leupa, aufgeteilt nach Krankheitsbildern. Gruppen A-K s. Text

- = Akute lymphatische Leukämie, Reticulose $0=$ Entzündliche Erkrankungen

$\square=$ Diabetes mellitus
$\Delta=$ Gesunde Kinder

geringfügig über dem Mittelwert der Gesunden (Harnwegsinfektion in Abheilung; unklarer Infekt im $\mathrm{Ab}$ klingen; eitrige Angina abklingend; Diabetes mit abklingendem Infekt; abgeheilte Pyelonephritis).

Dies gilt nur für Carboxypeptidase A (CNP). Die beiden anderen Enzymaktivitäten zeigen keinen sichtbaren Zusammenhang $z$ wischen gesundem und entzündlichem Serum. Alle Werte streuen gleich stark.

Im Kollektiv I (nicht abgebildet) liegen die Verhältnisse analog. Hohe Carboxypeptidase A-(CNP)-Aktivitäten finden sich bei den frischen Pneumonien $(2,7-3,9 \mathrm{mU} /$ $\mathrm{m} l$ ) und den allgemeinen bakteriellen Infektionen und gastrointestinalen Erkrankungen $(2,9-3,8 \mathrm{mU} / \mathrm{m} /)$. Abheilende Entzündungen (Pneumonie, Pleuropneumonie, Pyelonephritis, Carditis, Asthma) liegen wieder nahe dem Normalbereich, ebenso wie drei Fälle von chronischer myeloischer Leukämie. Die gesunden Kinder weisen Normalwerte auf. Die abheilenden Entzündungen beider Kollektive sind regelmäßig durch niedrige Blutkörperchensenkungsgeschwindigkeit gekennzeichnet. Die Erhöhung der Carboxypeptidase A (CNP) ist nicht sehr ausgeprägt. Der Mittelwert liegt bei 3,17 mU/ $\mathrm{m} l$, der Vertrauensbereich beträgt $16,5 \%$.

\section{Parallelität der Enqymaktivitäten}

Abbildung 4 zeigt die Korrelationen der drei Enzymaktivitäten zueinander, auf jeweils den Ordinaten aufgetragen. In keinem Fall ist eine Zuordnung oder eine Gruppierung um eine Gerade $\mathrm{x}-\mathrm{y}+\mathrm{a}=0$ zu erkennen. Die Aktivitäten sind voneinander unabhängig. Bereits an dieser Stelle sei auf die Frage der Spezifität der Carboxypeptidase A hingewiesen, da beide Substrate unterschiedlich gespalten werden.

\section{Enzymaktivitäten und Blutkörperchensenkungsgeschwindigkeit}

In Abbildung 5 und 6 ist der Zusammenhang zwischen den drei Enzymaktivitäten und der Blutkörperchen-

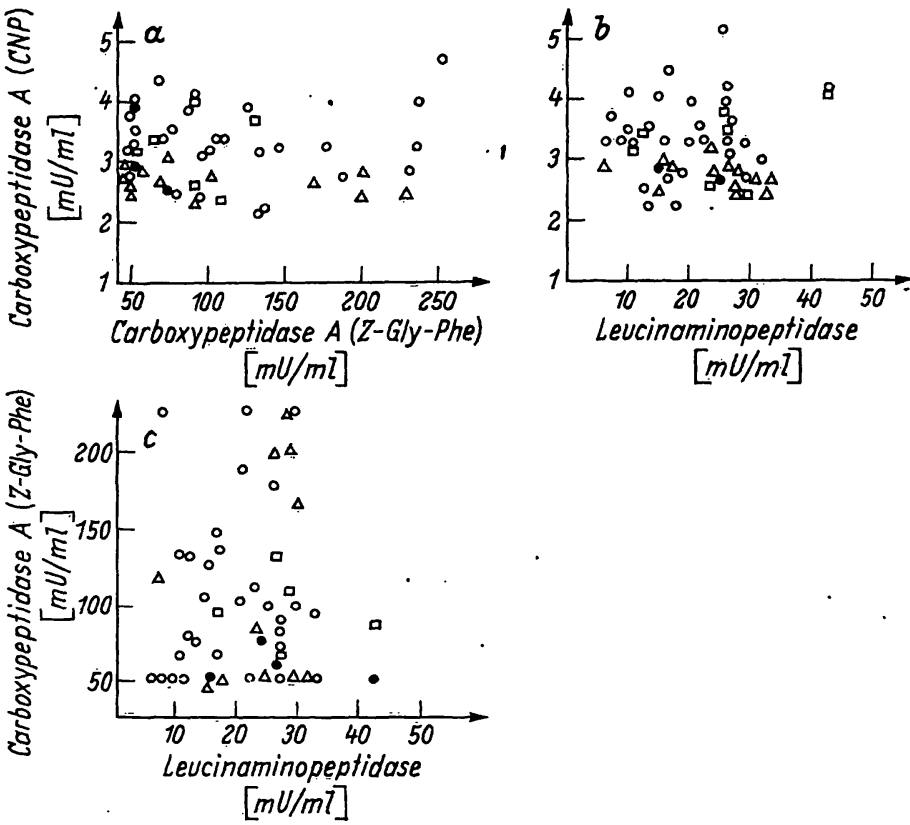

Abb. 4

Korrelation der Enzymaktivitäten gegenüber CNP, Z-Gly-Phe und

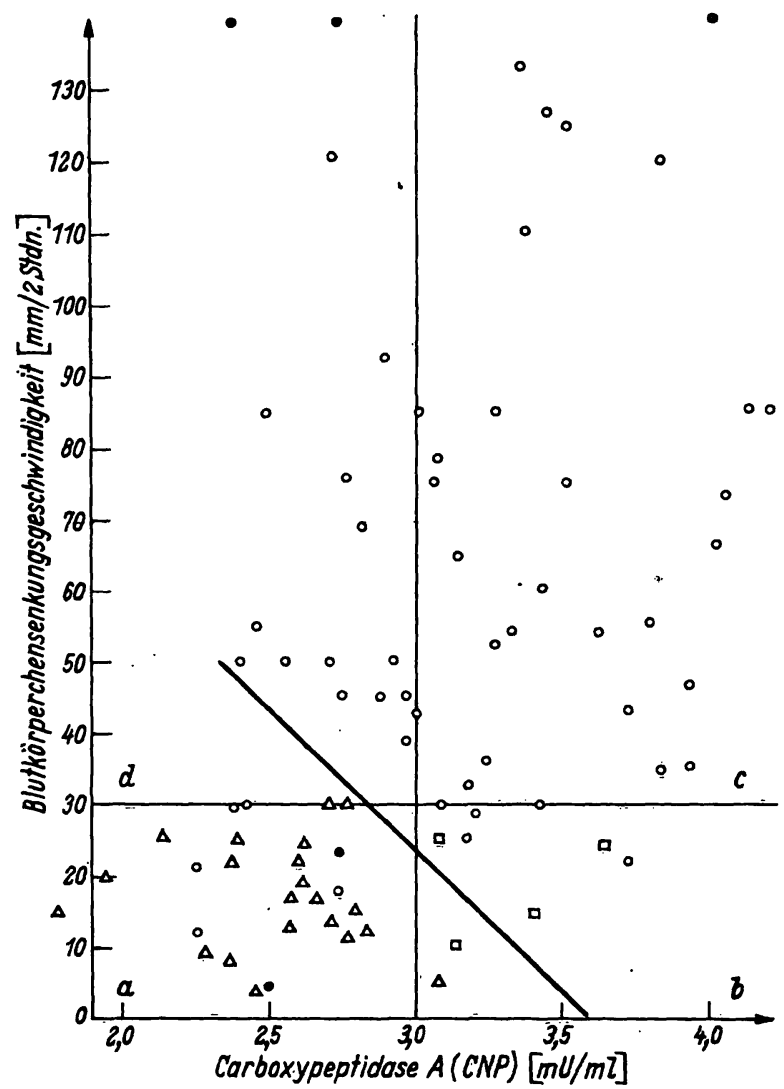

Abb. 5

Korrelation der Aktivität der Carboxypeptidase A gegenüber CNP und der Blutkörperchensenkungsgeschwindigkeit.

Quadrant a

niedere Blutkörperchensenkungsgeschwindigkeit, niedere Carboxypeptidase A (CNP), alle gesunden Kinder; Quadrant b

niedere Blutkörperchensenkungsgeschwindigkeit, hohe Carboxypeptidase A (CNP), u. a. Diabetes mit und ohne Infekt; Pseudocroup mit Lymphocytose; seröse Meningitis mit normalem Blutbild; Enteritis; Quadrant c

hohe Blutkörperchensenkungsgeschwindigkeit, hohe Carboxypeptidase $A$ (CNP), nur hochakute Entzündungen

Quadrant d

hohe Blutkörperchensenkungsgeschwindigkeit, niedere Carboxypeptidase $A$ (CNP) u. a. Beginnendes rheumatisches Fieber und $L y m p h$. ad enitis; eine größere Zahl abklingender Entzündungen wie Angina, Pleuropneumonien, Cárditis, Pyelonephritis sowie zwei Fälle chronischer lymphatischer Leukämie 


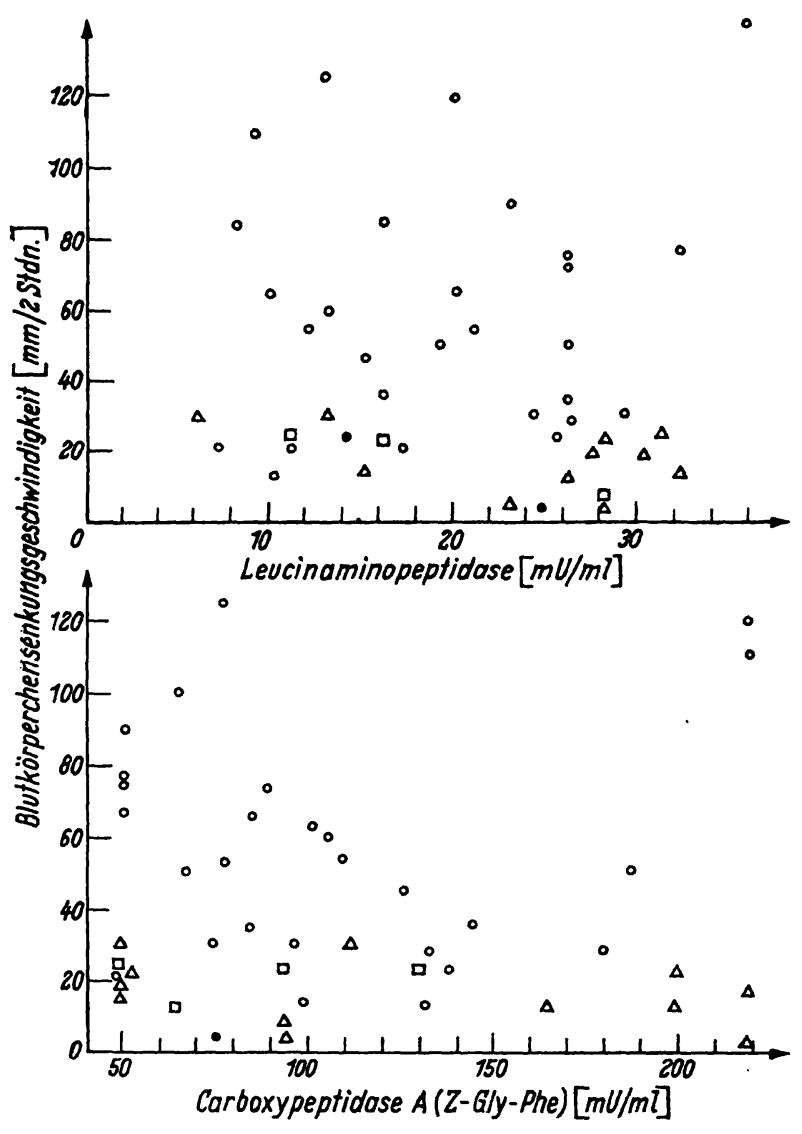

Abb. 6

Zusammenhang zwischen den Aktivitäten der Carboxypeptidase A gegenüber CNP und der ,Leucinaminopeptidase“ gegenüber Leupa und der Blutkörperchensenkungsgeschwindigkeit

senkungsgeschwindigkeit dargestellt. Abbildung 5 zeigt eine Korrelation: mit steigender Blutkörperchensenkungsgeschwindigkeit steigt prinzipiell auch die Aktivität der Carboxypeptidase $A$ (CNP) an. Das Bild wird klarer, wenn man die Seren zu vier Gruppen zusammenfaßt und in Quadranten aufteilt (vgl. Legende).

Die Grenze zwischen den gesunden und pathologischen Seren der Kollektive I + II liegt etwa bei der eingezeichneten Geraden $y=-a x+b$.

Carboxypeptidase A (Z-Gly-Phe) und Leucinaminopeptidase weisen keine Korrelation zur Blutkörperchensenkungsgeschwindigkeit auf (Abb. 6).

\section{Enzymaktivitäten und Leukozytenzabl}

Abbildung 7 demonstriert die Zusammenhänge zwischen der Aktivität der Carboxypeptidase A (CNP) und der absoluten Leukocytenzahl. Auch hier steigt die Enzymaktivität mit zunehmender Leukocytenzahl. Die Quadrantendarstellung zeigt ein der Abbildung 5 analoges Bild (vgl. Legende).

Wiederum läßt sich durch eine Gerade $y=-a x+b$ eine Grenze zwischen den gesunden und pathologischen Seren der Kollektive I + II ziehen.

Carboxypeptidase A (Z-Gly-Phe) und Leucinaminopeptidase zeigen keine Korrelation zur Leukocytenzahl; auf eine graphische Wiedergabe wurde. daher verzichtet.

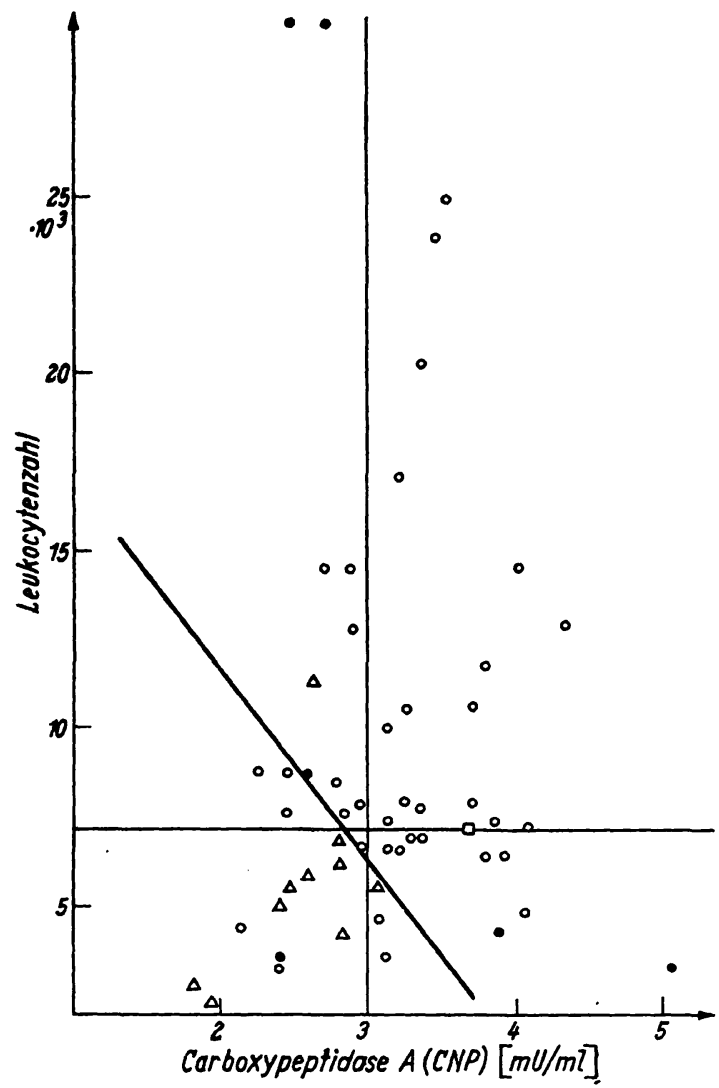

Abb. 7

Zusammenhang zwischen der Aktivität der Carboxypeptidase A gegenüber CNP und der absoluten Leukocytenzahl.

Quadrant a
niedere Leukocytenzahl, niedere Carboxypeptidase A (CNP), fast alle niedere Leukocytenzahl, niedere Carboxypeptidase A (CNP), fast alle
gesunden Fälle; fieberhafter Infekt mit Lymphocytose; Toxoplasmose mit Lymphocytose Quadrant b

niedere Leukocytenzahl, hohe Carboxypeptidase A (CNP), u. a. Pleuropneumonie; Diabetes mit Infekt; Pyelonephritis; seröse Meningitis; Mucoviscidose mit Pleuropneumonie;

hohe Leukocytenzahl, hohe Carboxypeptidase A (CNP), nur hochakute Entzündungen;

Quadrant d
hohe Leukocytenzahl, niedere Carboxypeptidase A (CNP), u. a. abklingende eitrige Angina; Carditis; Orbitalphlegmone; chronische Cystopyelitis mit Pneumonie; Lymphadenitis

\section{Diskussion}

In den letzten Jahren führten systematische Untersuchungen an der „Leucinaminopeptidase“ zur Auffindung einer neuen Enzymklasse, der Aminosäurearylamidasen. Diese hydrolysieren bevorzugt oder ausschließlich chromogene Modellsubstrate vom Typ der $\beta$-Naphthylamide und $p$-Nitranilide von neutralen, sauren oder basischen Aminosäuren oder Dipeptiden (4, 5, 8-14). Auf Grund der vorliegenden Befunde scheint ähnliches auch für Carboxypeptidasen zu gelten: die Hydrolyse des CNP ist wahrscheinlich auf Carboxypeptidasen zurückzuführen, die mit der klassischen Carboxypeptidase A nicht identisch sind. Von den in letzter Zeit beschriebenen Enzymen, saure und katheptische Carboxypeptidasen, Carboxytripeptidasen, Carboxypeptidasen $\mathrm{C}, \mathrm{N}, \mathrm{A}_{1}$ und $\mathrm{A}_{2}(4,15-23)$ könnten vor allem die Carboxypeptidase $N$ aus Humanserum, ein den Kininasen verwandtes Enzym oder die Carboxypeptidasen $A_{1}$ und $A_{2}$ aus Humanpankreas mit unserer Carboxypeptidase A (CNP) identisch sein. Jedenfalls 
steht erstmalig fest, daß eine Carboxypeptidase mit dem chromogenen Substrat CNP rasch, empfindlich und genau im Serum gemessen werden kann, selbst wenn damit nicht die "klassische" Carboxypeptidase A (EC 3.4.1.2) erfaßt wird - eine Schwierigkeit, der man sich bei der Verwendung des chromogenen Substrats Leupa für die Bestimmung der "Leucinaminopeptidase“ (EC 3.4.1.1) stets bewußt sein sollte.

Die Aktivitäten der Carboxypeptidase A (Z-Gly-Phe) und der Leucinaminopeptidase können für unser Problem unberücksichtigt bleiben, da sie keinen Bezug zum Krankheitstyp und -verlauf, zur Blutkörperchensenkungsgeschwindigkeit und zur Leukocytenzahl aufweisen. Für diagnostische Zwecke im Zusammenhang mit entzündlichen Prozessen ist ihre Bestimmung im Serum wertlos.

Die Aktivität der Carboxypeptidase A (CNP) ist der Blutkörperchensenkungsgeschwindigkeit und der Leukocytenzahl bei praktisch allen akuten oder schweren Entzündungsbildern signifikant korreliert: niederen Werten der Blutkörperchensenkungsgeschwindigkeit und Leukocytenzahl stehen niedere Aktivitäten (Gesunde), erhöhten Werten der Blutkörperchensenkungsgeschwindigkeit und Leukocytenzahl erhöhte Aktivitäten (akute hochentzündliche Prozesse) gegenüber (Quadranten a und $c$ der Abb. 5 und 7).

Bei der Regressionsanalyse wurden $(p<0,05)$ Regressionskoeffizienten $b=319,66$ bzw. 347,49 , Korrelationskoeffizienten $\mathrm{r}=0,55$ bzw. 0,41 sowie ein $\mathrm{Be}$ stimmtheitsma $B=0,31$ bzw. 0,17 errechnet. Dies deutet darauf hin, daß die Carboxypeptidase A (CNP) - im Gegensatz zur Carboxypeptidase A (Z-Gly-Phe) und Leucinaminopeptidase - aus den Leukocyten stammt und somit lysosomalen Ursprungs ist, während letztere gewebslokalisiert zu sein scheinen und bei Entzündungsvorgängen nicht oder nur bei Beteiligung der betroffenen Organe in die Blutbahn abgegeben werden. Differentialdiagnostisch interessant sind die Fälle, bei denen die obengenannten Korrelationen nicht gegeben sind. Wir vermögen den Typ rlicht zu deuten, bei dem eine erhöhte Enzymaktivität bei niederer Blutkörperchensenkungsgeschwindigkeit und Leukocytenzahl auftritt (Quadrant b). Wichtig erscheint uns, daß abklingende entzündliche Prozesse klinisch-chemisch möglicherweise faßbar werden, indem bei noch erhöhter Blutkörperchensenkungsgeschwindigkeit und Leukocytenzahl die Carboxypeptidase A (CNP) bereits wieder absinkt (Quadrant b); prognostisch wäre dies bedeutungsvoll. Möglicherweise tritt das gleiche Bild auch beim Beginn solcher Erkrankungen auf, was diagnostisch auf Grund der rasch manifest werdenden Blutkörperchensenkungsgeschwindigkeits-Erhöhung weniger bedeutsam wäre.

Wir möchten ausdrücklich darauf hinweisen, daß u. E. das absolute Ausmaß der Aktivitätserhöhung gering und die Fallzahl für die einzelnen Krankheitsbilder recht klein ist und $\mathrm{da} a$ außerdem alle Befunde nut an Kindern erhoben wurden. Wir sind trotzdem der Auffassung, daß das Substrat $\mathrm{N}_{\alpha}$-Carbonaphthoxy-L-phenylalanin zur raschen, empfindlichen und exakten Bestimmung einer Carboxypeptidase in Humanseren geeignet ist und das Verhalten dieses Enzyms, besonders bei entzündlichen Krankheitsprozessen, in größerem Maßstab überprüft werden sollte. Bei Bestätigung unserer Befunde sollten diese unter pathophysiologischen und diagnostischen Gesichtspunkten diskutiert werden.

Wir danken Herm KehrhaHN, Fa. Knoll A.-G., für die Hilfe bei der statistischen Auswertung. Besonderer Dank gebührt auch Frl. WEBER, chem. techn. Ass. Fa. Knoll A.-G., für die gewissenhafte, selbständige Mitarbeit bei den zugrundeliegenden wissenschaftlichen Vorarbeiten.

\section{Literatur}

1. Heister, R. und H. F. HofmanN, „Die Entzündung“, Grundlagen und pharmakologische Beeinflussung, 1. Aufl., Urban \& Schwarzenberg, München-Berlin-Wien (1966). - 2. Missmaki, H. P. und G. Riethmüller, Blut 15, 198 (1967). - 3. Apper, W. und E. Huth, in Vorbereitung. - 4. AppeL, W., in: H. U. BergMEYER, Methoden der enzymatischen Analyse, 2. Aufl., Verlag Chemie, Weinheim/Bergstr., in Vorbereitung. - 5. WILLIG, F., I. Greiner, H. Stork und F. H. Schmidt, Klin. Wschr. 15, 474 (1967). - 6. Folk, I. E. und I. A. Gladner, J. biol. Chemistry 235, 60 (1960). - 7. SCHÖN, H., B. RässLER und N. HENNING, Klin. Wschr. 39, 217 (1961). - 8. Wüst, H., in: H. U. BerGMEYER, Methoden der enzymatischen Analyse, 1. Aufl., Verlag Chemie, Weinheim/Bergstr. (1962). - 9. MäKINEN, P. L. und I. Raekallio, Acta chem. Scand. 21, 761 (1967) und 22, 597 (1968). - 10. MäKInEN, K. K., Arch. Biochem. Biophysics 126, 803 (1968). - 11. MCDonald, J. K., F. H. LeIBaCh, R. E. GrinDeland und St. Ellis, J. biol. Chemistry 243, 4143 (1968). -
12. Marks, N., R. K. Datta und A. Lajtha, J. biol. Chemistry 243, 2882 (1968). - 13. RINNE, U. K. und P. RiEKKINEN, Acta Neurol. Scand. 44, 156 (1968). - 14. Behal, F. J., G. H. Littue und R. A. KleIN, Biochem. biophysica Acta (Amsterdam) 178, 118 (1969). - 15. Stein, U. und D. Platt, Klin. Wschr. 46, 1145 (1968). - 16. Stein, U., U. Weber und E. Buddecke, HoppeSeyler's Z. physiol. Chem. 349, 472 (1968). - 17. Nordwig, A., Hoppe-Seyler's Z. physiol. Chem. 349, $1353^{\circ}$ (1968). - 18. ZuBER, H., Hoppe-Seyler's Z. physiol. Chem. 349, 1337 (1968). 19. Johnson, D. C. und J. W. Ryan, Biochem. biophysica Acta (Amsterdam) 160, 196 (1968). - 20. Trapesnikova, S. S. und T. S. Paskrina, Biokhimiya (Moskau) 33, 1012 (1968). 21. YANG, H. Y. T., E. G. ERdös und T. S. ChIANG, Nature (London) 218, 1224 (1968). - 22. Ebata, M. und K. MiYazAKI, Experientia Basel 23, 1007 (1967). - 23. HADORN, B. und V. L. SilberberG, Biochim. biophysica Acta (Amsterdam) 151, 702 (1968).
Dr. W. Appel

Knoll A.-G.

6700 Ludwigshafen/Rh. 2 